\title{
Reclassification and treatment of odontogenic keratocysts: A cohort study
}

\author{
Ophir RIBEIRO-JÚNIOR ${ }^{(a)}$ \\ Alexandre Meireles BORBA(a) \\ Carlos Augusto Ferreira ALVES(b) \\ Marcia Maria de GOUVEIA(b) \\ Maria Cristina Zindel DEBONI(a) \\ Maria da Graça \\ NACLÉRIO-HOMEM(a) \\ (a) Universidade de São Paulo - USP, School \\ of Dentistry, Department of Oral \& \\ Maxillofacial Surgery, Traumatology and \\ Prosthesis, São Paulo, SP, Brazil. \\ (b) Universidade de São Paulo - USP, \\ University Hospital, Department of Oral \& \\ Maxillofacial Surgery, São Paulo, SP, Brazil.
}

Declaration of Interest: The authors certify that they have no commercial or associative interest that represents a conflict of interest in connection with the manuscript.

Correspondending Author:

Ophir Ribeiro Júnior

E-mail: ophirri@ig.com.br

https://doi.org/10.1590/1807-3107bor-2017.vol31.0098

Submitted: June 06, 2017

Accepted for publication: Sep 27, 2017

Last revision: Oct 19, 2017
Abstract: The odontogenic keratocyst $(\mathrm{OKC})$ is a recurrent cyst that has been recently reclassified from an odontogenic tumor to an odontogenic cyst. The aim of the present study was to investigate its treatment and address issues related to its association with nevoid basal cell carcinoma syndrome (NBCCS). Lesions from the cohort of patients included in the present study consisted of 40 OKCs, of which 27 lesions were treated by enucleation (GE) and 13 underwent decompression (GD). Complementary treatment occurred in 38 (95\%) lesions, of which 10 underwent isolated peripheral ostectomy (GO) and 28 underwent peripheral ostectomy combined with Carnoy's solution (GC). Thirteen lesions were associated with NBCCS (GS), while the others $(n=27)$ were non-syndromic lesions (GnS). The recurrence-free periods (RFP) in the sample groups were compared using the Kaplan-Meier function and log-rank test at a significance level of 5\% $(p<0.05)$ and were used to calculate the cumulative risk of recurrence (CRR) in each postoperative year. During the follow-up period, which had a mean of 43.5 months (range: 12-102 months), six (15\%) recurrences were diagnosed. There was no significant difference among the RFP for the compared groups $(p>0.05)$ or increased CRR for the decompression (15.4\%) over five years. Application of Carnoy's solution did not increase the efficacy of the peripheral ostectomy, but was related to a CRR of $0 \%$ for the syndromic lesions over five years. Therefore, 1) decompression did not increase the recurrence risk; 2) peripheral ostectomy demonstrated a similar efficacy as the combination with Carnoy's solution;3) the association of NBCCS did not seem to significantly influence OKC recurrence; and 4) syndromic lesions seem to behave in the same manner as non-syndromic lesions when submitted to complementary treatments.

Keywords: Odontogenic cyst; Recurrence; Surgery, Oral.

\section{Introduction}

The odontogenic keratocyst $(\mathrm{OKC})$ is a controversial odontogenic developmental cyst that has undergone conceptual and terminological changes in recent decades. ${ }^{1,2,3,4,5}$ The name "keratocyst" arose in the 1950s to describe any cyst that exhibited keratinization and was recommended as the official terminology for a specific type of odontogenic cyst in the World Health Organization (WHO) classifications of 1971 and 1992. ${ }^{6,7}$ There were two histopathological subtypes of OKC: parakeratinized and orthokeratinized. ${ }^{8,9}$ 
In the following years, there was much discussion about the need for distinguishing the two subtypes of OKC from one another since the parakeratinized one presented a higher recurrence rate and an eventual association with nevoid basal cell carcinoma syndrome (NBCCS). This evidence resulted in the separation of these subtypes into two distinct diseases and the abandonment of the term "OKC" by the WHO in 2005. ${ }^{10}$ The parakeratinized subtype became the "keratocystic odontogenic tumor" (KCOT), which integrated the group of odontogenic epithelial tumors into its classification, while the orthokeratinized subtype continued in the group of odontogenic developmental cysts as "orthokeratinized odontogenic cyst".

Recently in 2017, the KCOT returned to the WHO classification of odontogenic developmental cysts, retaking the original terminology "OKC" ${ }^{11}$ In the current classification, the new OKC remains distinct from the orthokeratinized odontogenic cyst and maintains the histopathological criteria that were established in 2005: a parakeratinized epithelial lining, five to eight layers, and may present with areas of squamous metaplasia when there is inflammation in the capsule.

The treatment for OKC remains controversial, but the return to the odontogenic cysts group stimulates a trend of conservative methods, such as decompression and enucleation with complementary techniques. ${ }^{12,13,14,15,16}$ Decompression minimizes tissue damage adjacent to the OKC, although its effectiveness in controlling recurrence is questioned. ${ }^{17,18,19}$ Among the complementary treatments, peripheral ostectomy is emphasized as a technique that can be easily performed by conventional surgical armamentarium and that has a low complication rate. ${ }^{5}$ Its combination with the use of Carnoy's solution is efficient, although the risk for complications might be increased by doing so. ${ }^{20,21,22}$

The benefits of complementary treatment are relevant, but there is insufficient evidence to prove its efficacy for OKCs associated with NBCCS, which have a theoretical higher risk of recurrence. ${ }^{19} \mathrm{~A}$ lack of studies on the treatment of OKC associated with NBCCS has been confirmed in a recent systematic review, ${ }^{23}$ which indicates that minimal evidence exists on this specific type of OKC. Therefore, the evaluation of treatments for OKCs associated with NBCCS is necessary.
The study of OKC and other recurrent cysts and tumors can be enhanced by analyzing the diseasefree or recurrence-free period, allowing for the determination of the cumulative risk for the recurrence for every year of follow-up and a comparison among different treatments. Such a methodology is commonly used in the oncology field and was applied in the present study.

The aim of this paper was to answer the following questions:

a. Does the decompression technique increase the risk of recurrence?

b. Does the peripheral ostectomy technique have a higher efficacy when it is used in combination with Carnoy's solution?

c. Does the OKC association with NSCCS influence the recurrent rate?

d. Would enucleation with complementary treatments provide different outcomes when used in NBCCS-associated lesions?

\section{Methodology}

The OKCs treated between 2003 and 2009 at the University Hospital and the Faculty of Dentistry of the University of São Paulo (USP) were retrospectively evaluated in this cohort study to investigate the influence of treatments and NBCCS on the incidence of recurrences. This study followed the STROBE guidelines and was performed in accordance with the principles of the Declaration of Helsinki, and the Ethics Committee of the USP provided ethical approval for the proposed research project under the protocol number 65/2009.

The eligibility of the OKCs was determined by OKC histological diagnosis confirmation according to the WHO recommendation, ${ }^{10,11}$ and the eligible lesions included those associated or not associated with NBCCS that had a minimum of 12 months of follow-up after any of the respective treatments: enucleation, decompression and peripheral ostectomy alone or in combination with the application of Carnoy's solution. The author ORJ participated in all of the treatments as the main or auxiliary surgeon.

The treatments were carried out according to the following technical criteria and aspects: 
a. Enucleation - performed by detachment of the cyst from the bone cavity, either directly or after decompression (Figure 1).

b. Decompression - performed as marsupialization or with the use of a temporary decompression device (Figure 2). The duration of this therapy was calculated from the initial decompressive maneuver until the day of enucleation of the lesion or when its complete involution was determined in the clinical and radiological exams (without any surgical removal).

c. Peripheral ostectomy - performed in the bone cavity, after enucleation, and with the use of round burs and conspicuous saline irrigation (Figure 3). This method was used in all of the enucleated lesions that received complementary treatment of the bone cavity, either alone or in

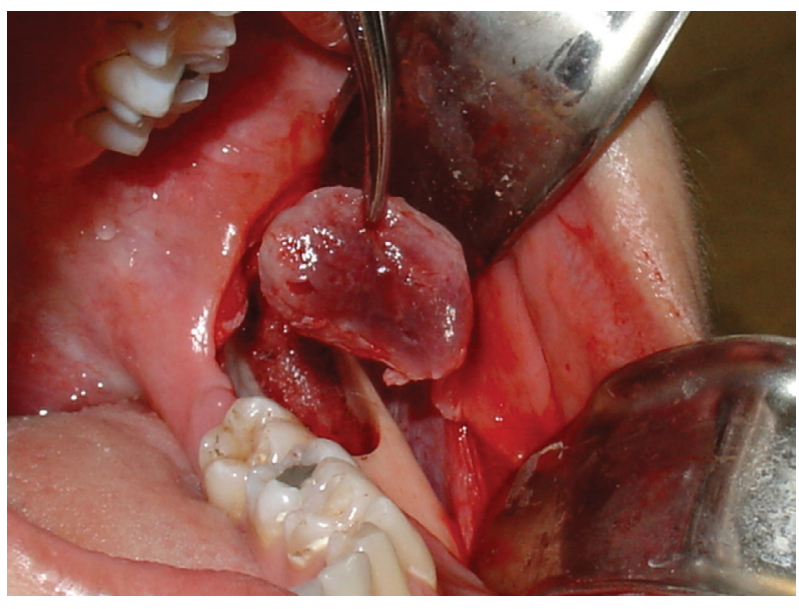

Figure 1. Enucleation.

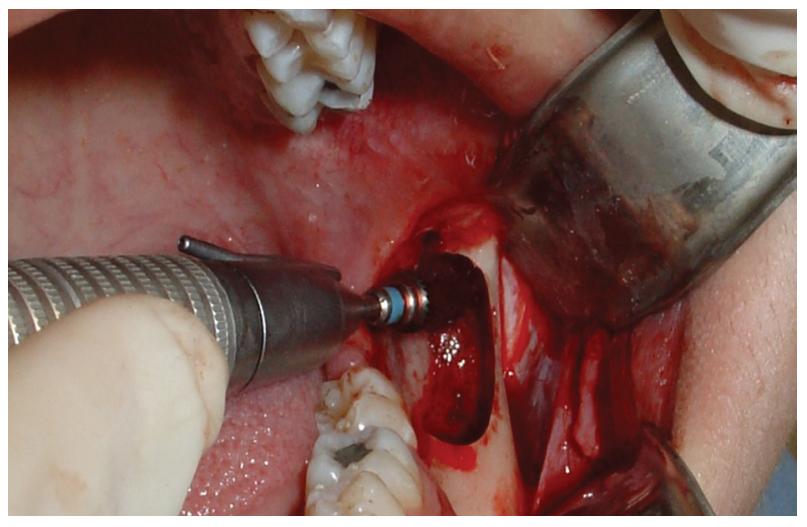

Figure 3. Peripheral ostectomy. combination with Carnoy's solution.

d. Carnoy's solution - each $10 \mathrm{ml}$ of this solution contained $6 \mathrm{ml}$ of $95 \%$ ethanol, $3 \mathrm{ml}$ of chloroform, $1 \mathrm{ml}$ of glacial acetic acid and $1 \mathrm{~g}$ of ferric chloride. The solution was applied by soaking sterile gauze with Carnoy's solution in the bone cavity after peripheral ostectomy for three minutes followed by conspicuous saline irrigation (Figure 4).

Enucleation and decompression were classified as the main treatments, while isolated peripheral ostectomy and the supplemental combination with the application of Carnoy's solution represented the complementary treatments.

Through the medical records of the patients with the lesions, the OKC lesions were classified as syndromic or non-syndromic according to their association with NBCCS. The diagnosis of the

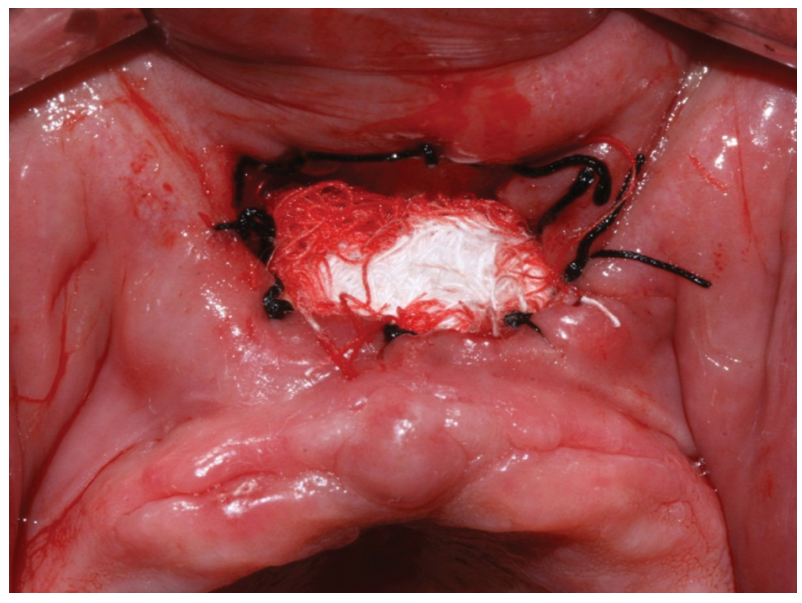

Figure 2. Decompression (using the marsupialization technique).

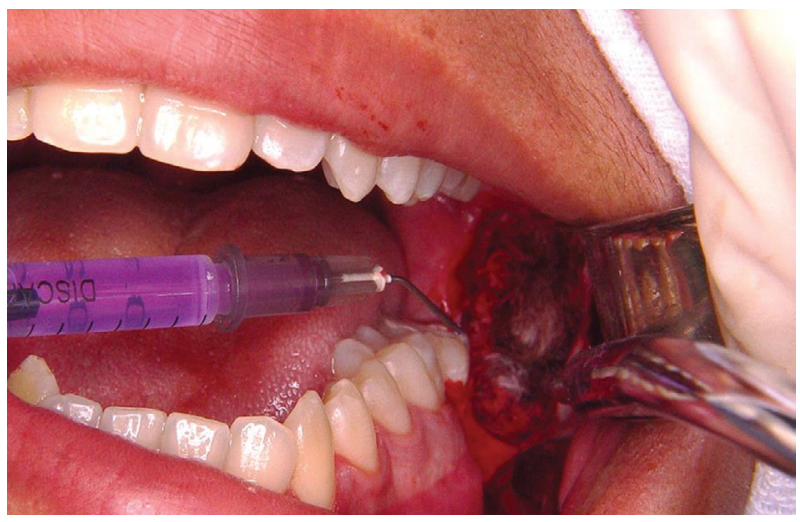

Figure 4. The application of Carnoy's solution. 
syndrome was considered in subjects who presented with OKCs and another major criterion or two minor criteria, which were established by Evans et al. ${ }^{24}$

\section{Analysis of the recurrence-free period}

To analyze the recurrence-free period (RFP), the lesions were separated into groups according to the following variables: main treatments, complementary treatments and NBCCS. The main treatment variables were defined by lesions submitted to only enucleation (GE) and lesions that underwent decompression with or without further enucleation (GD). The complementary treatment variables consisted of lesions that were submitted to only peripheral ostectomy (GO) and those submitted to peripheral ostectomy followed by the use of Carnoy's solution (GC). To verify the influence of NBCCS, the variables were lesions syndromic (GS) e not syndromic (GnS). The last variables were syndromic (GS2) and non-syndromic (GnS2) lesions treated by direct enucleation (without decompression) and peripheral ostectomy in combination with Carnoy's solution.

The follow-up period was established as a duration of months and was determined by the interval between the treatment and the most recent consultation, which consisted of physical and radiographic evaluations (panoramic radiographs for all of the cases and CT scans for cases in which an anatomic superimposition to other structures impaired a simple radiographic evaluation).

The RFP was calculated until the last follow-up for non-recurrent lesions and until the diagnosis of recurrence for recurrent lesions. The recurrence criteria were based on the presence of a radiographic or tomographic image suggesting a new lesion within the boundaries of the original one, and confirmation by histopathological diagnosis of OKC was made in the cases of recurrence.

An estimate of the RFP was obtained after the decompression for GD and enucleation for GE, GO and GC. Although GO and GC also underwent decompression, the decompression period was not considered when estimating the RFP since the complementary treatment. Conversely, decompressed lesions among GS and GnS also underwent analysis from the moment of decompression.
The SPSS software 15.0 was used for the statistical analysis. The RFP was compared using the log-rank test and was used to estimate the cumulative risk of recurrence (CRR) and construction of the graphics by the Kaplan-Meier analysis. The censure criterion was defined by the absence of recurrence, regardless of the time of the follow-up, and censured lesions contributed to the graphics until the last follow-up. The statistical tests were performed at a significance level of $5 \%$.

\section{Results}

A total of 40 lesions were obtained from 31 subjects (18 men and 13 women) with an age range of 9 to 71 years (mean: 33.3 years), including 4 subjects who were diagnosed with NBCCS. Most of the lesions occurred at the mandible $(n=27 ; 67.5 \%)$ with a mandibular ramus prevalence and displaying an unilocular pattern $(\mathrm{n}=35 ; 87.5 \%)$. Thirteen lesions (32.5\%) were classified as syndromic.

Twenty-seven (67.5\%) lesions received direct enucleation and composed the GE, while the remainder of the lesions in the total sample $(n=13 ; 32.5 \%)$, which were submitted to decompression, formed the GD. Complementary treatments did not occur in two children whose lesions comprised the GD, including one lesion that totally regressed and another that presented adjacent teeth in the process of eruption. Of the 38 lesions that received complementary treatment, ten $(26.3 \%)$ were treated with peripheral ostectomy and composed the GO, while the other 28 (73.7\%), which also received Carnoy's solution, formed the GC. Regarding the association with NBCCS, 13 (32.5\%) lesions were classified in GS and 27 $(67.5 \%)$ in GnS. Direct enucleation and complementary treatments (peripheral ostectomy and application of Carnoy's solution) determined 10 lesions in GS2 and 11 in GnS2.

The recurrences in each sample group are shown in Table 1. Six (15\%) of the 40 lesions recurred in a follow-up period ranging from 12 and 102 months (mean: 43.5 months), and the time for diagnosis of each recurrence varied from 18 to 76 months (mean: 33.5 months). Among the main treatments, GE and GD exhibited recurrences of $14.8 \%$ and $15.4 \%$, respectively, while GO presented a recurrence rate of $10 \%$ and its 
Table 1. The recurrence (\%) and estimation of the recurrence-free period by the Kaplan-Meier function is summarized with a p-value reported for the comparisons between groups.

\begin{tabular}{|c|c|c|c|c|c|c|c|}
\hline \multirow{3}{*}{ Variable } & \multirow{3}{*}{$\mathrm{n}$} & \multicolumn{2}{|c|}{ Recurrence } & \multicolumn{4}{|c|}{ Recurrence-free period in months } \\
\hline & & \multirow{2}{*}{$\mathrm{n}$} & \multirow{2}{*}{$(\%)$} & \multirow{2}{*}{ Average } & \multicolumn{2}{|c|}{$\mathrm{Cl}(95 \%)$} & \multirow{2}{*}{$p$} \\
\hline & & & & & Inferior & Superior & \\
\hline Main treatment & & & & & & & 0,905 \\
\hline Enucleation (GE) & 27 & 4 & 14.8 & 77.8 & 65.4 & 90.2 & \\
\hline Decompression (GD) & 13 & 2 & 15.4 & 63.8 & 53.4 & 74.2 & \\
\hline Complementary treatments & & & & & & & 0,662 \\
\hline Peripheral ostectomy (GO) & 10 & 1 & 10.0 & 34.0 & 30.4 & 37.6 & \\
\hline $\begin{array}{l}\text { Peripheral ostectomy + } \\
\text { Carnoy's solution (GC) }\end{array}$ & 28 & 4 & 14.3 & 79.8 & 69.0 & 90.6 & \\
\hline NBCCS & & & & & & & 0,833 \\
\hline Syndromic (GS) & 13 & 2 & 15.4 & 80.2 & 63.7 & 96.7 & \\
\hline Non-syndromic (GnS) & 27 & 4 & 14.8 & 65.7 & 56.5 & 74.8 & \\
\hline \multicolumn{4}{|c|}{ NBCCS: enucleation + complementary treatments } & & & & 0,391 \\
\hline Syndromic (GS2) & 10 & 1 & 10.0 & 88.8 & 83.2 & 94.4 & \\
\hline Non-syndromic (GnS2) & 11 & 2 & 18.2 & 64.9 & 51.5 & 78.2 & \\
\hline
\end{tabular}

$\mathrm{n}$ : number; $\mathrm{Cl}$ : confidence interval; NBCCS: nevoid basal cell carcinoma syndrome.

combination with Carnoy's solution (GC) presented a recurrence rate of $14.3 \%$. The syndromic (GS) and non-syndromic $(\mathrm{GnS})$ lesions had similar recurrence rates, with respective values of $15.4 \%$ and $14.8 \%$. When only enucleation and complementary treatments were observed, the recurrence was lower for the syndromic lesions (GS2), which had a rate of $10 \%$.

In the calculation of the RFP, four lesions from four subjects were censured with the minimum follow-up time of 12 months. The follow-up losses occurred due to address change in one case, refuse to follow-up in two cases and death of the patient in one case. The recorded death involved one of the NBCCS patients and had no relation to any of the components of the syndrome or to the treatment instituted. The data related to the calculation of the RFP are described in Table 1 and Figures 5 to 8.

The main treatment, complementary treatment and association with NBCCS did not affect the RFP since there was no significant difference in these parameters among the groups of lesions ( $p>0.05)$. The Kaplan-Meier analysis estimated the RFP using the mean period (expressed as the number of months of treatment) for each variable. The main treatment variables presented RFP values of 77.8 and 63.8 (GE and GD, respectively), while the complementary treatment variables exhibited RFP values of 34 and 79.8 (GO and GC, respectively). The NBCCS variable had RFP values of 80.2 and 65.7 in the total sample (for GS and GnS, respectively) and RFP values of 88.8 and 64.9 in lesions submitted to direct enucleation (without decompression) and complementary treatments (GS2 and GnS2, respectively).

The CRR reached a maximum percentage during the first three years of the five years that were evaluated for all of the groups (Table 2). For the main treatment, the CRR from the $3^{\text {rd }}$ year to the $5^{\text {th }}$ year was $19.2 \%$ for enucleation and $15.4 \%$ for decompression (GD). For the complementary treatment, peripheral ostectomy (GO) reached a CRR of $16.7 \%$ at the end of the $3^{\text {rd }}$ year and the CRR of its combination with the use of Carnoy's solution was 15.3\% from the $3^{\text {rd }}$ year to the $5^{\text {th }}$ year. The syndromic lesions (GS) displayed a lower CRR from the $3^{\text {rd }}$ to the $5^{\text {th }}$ year than the non-syndromic lesions (GnS). The CRR for the syndromic lesions was $12.5 \%$, while that of the non-syndromic lesions was $21.3 \%$. The same pattern was observed for the effect of NBCCS on lesions submitted to only direct enucleation (without decompression) and those with complementary treatments (GS2), with a CRR of $0.0 \%$ until the end of the $5^{\text {th }}$ year. Due to the onset of a recurrence at the seventh year, there was a late increase in the risk of recurrence for the GE, GC, GS and GS2 groups, as displayed by the graphics of the Kaplan-Meier analysis (Figures 5-8). 


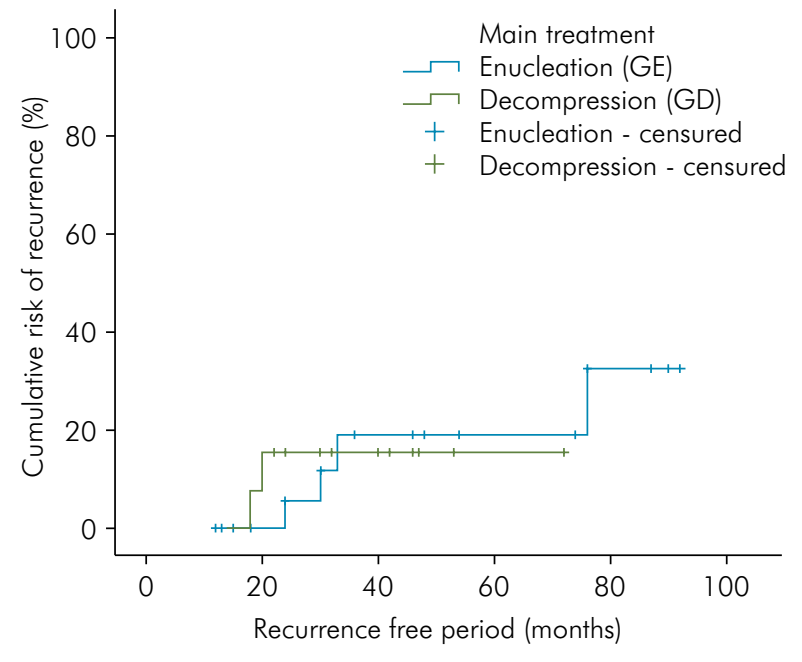

Figure 5. The cumulative risk of recurrence by the Kaplan-Meier analysis according to the main treatments.

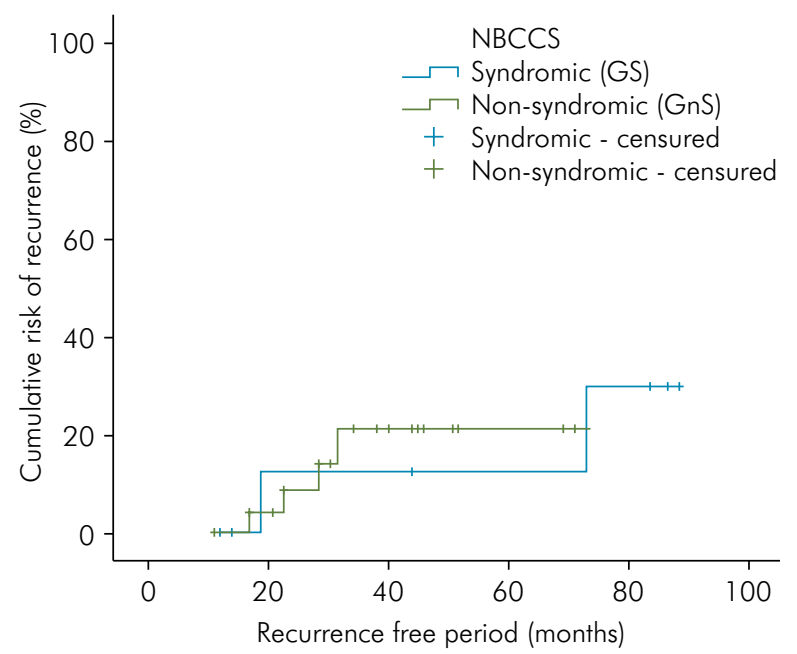

Figure 7. The cumulative risk of recurrence by the Kaplan-Meier analysis according to the association or lack thereof with the nevoid basal cell carcinoma syndrome.

\section{Discussion}

Treatment for $\mathrm{OKC}$, which varies from simple enucleation and decompression to resection, still poses dilemmas. ${ }^{1,2,3,4,5,10}$ The high recurrence rates published in the 70s required an understanding of the recurrence mechanism and, consequently, an improvement of the treatment techniques. Complementary methods, such as excision of the adjacent mucosa, peripheral ostectomy, cryotherapy and application of Carnoy's solution, were later addressed in the management of OKCs after

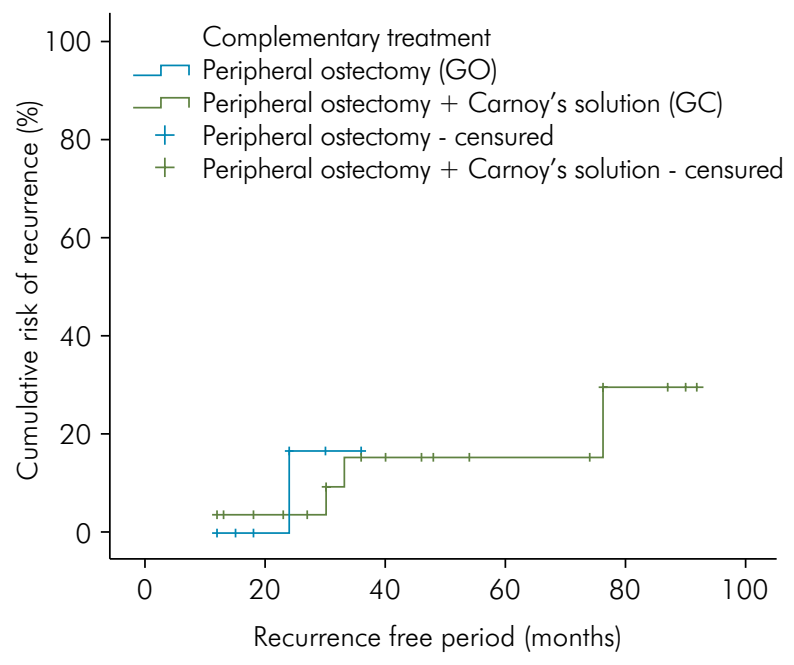

Figure 6. The cumulative risk of recurrence by the Kaplan-Meier analysis according to the complementary treatments.

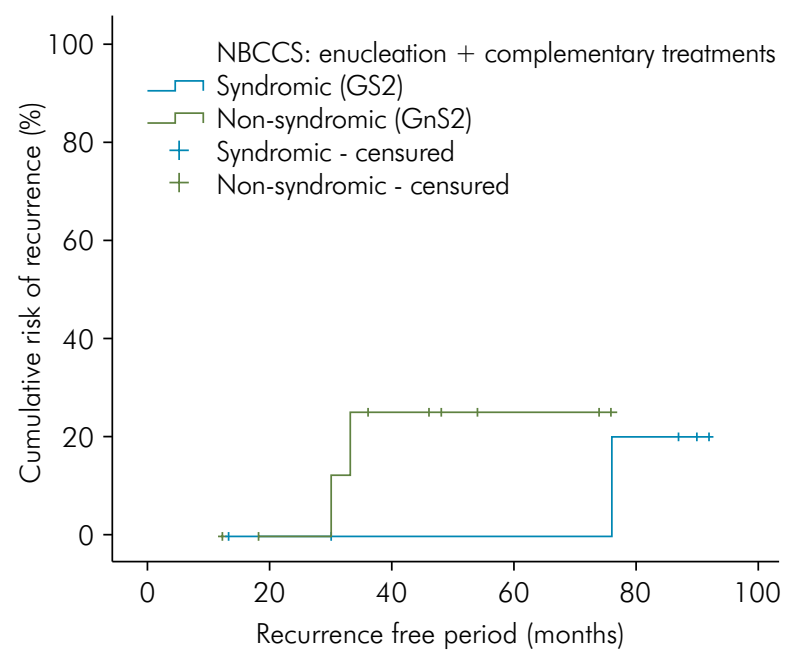

Figure 8. The cumulative risk of recurrence by the Kaplan-Meier analysis according to the association or lack thereof with the nevoid basal cell carcinoma syndrome for enucleation complemented by peripheral ostectomy and the use of Carnoy's solution.

its enucleation, minimizing the recurrence rates. ${ }^{25,26}$ Resection, which was already a treatment option, gained more prominence when OKC was classified as an odontogenic tumor. ${ }^{19,27,28}$ The reclassification of $\mathrm{OKC}$ as an odontogenic cyst stimulates the choice of conservative treatments and reinforces the continuity of investigations on decompression and complementary treatments, which was done in this study.

Despite the reclassification, all 40 lesions analyzed in the present study had a parakeratinized epithelial 
Table 2. The cumulative risks of recurrence (\%) in the sample groups are summarized.

\begin{tabular}{|c|c|c|c|c|c|c|}
\hline \multirow{2}{*}{ Variable } & \multirow{2}{*}{$\mathrm{n}$} & \multicolumn{5}{|c|}{ Cumulative risk of recurrence (\%) by year } \\
\hline & & 1st year & 2nd year & 3rd year & 4th year & 5 th year \\
\hline \multicolumn{7}{|l|}{ Main treatment } \\
\hline Enucleation (GE) & 27 & 0.0 & 5.6 & 19.2 & 19.2 & 19.2 \\
\hline Decompression (GD) & 13 & 0.0 & 15.4 & 15.4 & 15.4 & 15.4 \\
\hline \multicolumn{7}{|l|}{ Complementary treatment } \\
\hline Peripheral ostectomy (GO) & 10 & 0.0 & 16.7 & 16.7 & - & - \\
\hline Peripheral ostectomy + Carnoy's solution (GC) & 28 & 3.6 & 3.6 & 15.3 & 15.3 & 15.3 \\
\hline \multicolumn{7}{|l|}{ NBCCS } \\
\hline Syndromic (GS) & 13 & 0.0 & 12.5 & 12.5 & 12.5 & 12.5 \\
\hline Non-syndromic (GnS) & 27 & 0.0 & 8.7 & 21.3 & 21.3 & 21.3 \\
\hline \multicolumn{7}{|l|}{ NBCCS: enucleation + complementary treatments } \\
\hline Syndromic (GS2) & 10 & 0.0 & 0.0 & 0.0 & 0.0 & 0.0 \\
\hline Non-syndromic (GnS2) & 11 & 0.0 & 0.0 & 25.0 & 25.0 & 25.0 \\
\hline
\end{tabular}

n: number; NBCCS: nevoid basal cell carcinoma syndrome.

lining and met the histological criteria recommended by the WHO classification for the diagnosis of OKC.11 As in many studies on OKC, the groups presented here consisted of lesions instead of subjects. ${ }^{13,14,16,29,30}$ This methodology was more appropriate for determining the OKC RFP among subjects diagnosed with NBCCS because multifocal lesions in the same patient might undergo distinct surgical therapies at different moments.

The total number of lesions included in the cohort was defined by the subjects who entered the study period. Therefore, there was no previous definition of a sample size calculation. All of the non-recurrent lesions contributed to the RFP analysis with the maximum follow-up time after treatment and were denominated as censured as they left the RFP analysis.

The Kaplan-Meier analysis enabled for the comparison of therapies that presented with divergences related to the follow-up of non-recurrent lesions, such as those for GO and GC. The divergence between the means of the RFP in the complementary treatments (Table 1) was a reflection of the shorter follow-up period in the GO, but this did not compromise the statistical analysis because the comparison occurred in the period in which the curves of the Kaplan-Meier graph coincided; that is, in the first three years (Figure 6).

Others have used the Kaplan-Meier analysis and demonstrated a CRR of 35\% within the first five years for lesions that are not associated with NBCCS and that underwent enucleation without complementary treatment. ${ }^{31}$ The present research calculated the CRR for a five-year period and showed lower values for decompression and syndromic association (15.4\% and $12.5 \%$, respectively) in a sample characterized by the frequent use of complementary treatments (Table 2).

Despite the controversies related to the decompression of OKCs, studies have demonstrated that such a method produces recurrences equivalent to or even lower than those of direct enucleation. $12,14,15,16,28$ The recurrence after decompression $(15.4 \%)$ was similar to that after direct enucleation (14.8\%), with no significant difference in the RFPs and with a lower CRR (Tables 1 and 2). These results showed that decompression did not increase the risk for recurrence in the medium term.

Some articles report a recurrence greater than $30 \%$ when decompressed OKCs did not undergo further enucleation, and this percentage might be related to the presence of residual cystic epithelium and possible satellite microcysts. ${ }^{15,17,18,28}$ The sample presented here could not be evaluated for decompression without further enucleation because only one OKC did not undergo further surgery.

The use of Carnoy's solution in combination with peripheral ostectomy may increase its safety margins and compensate for its deficiency when lesions are near soft tissues and between dental roots. Chow ${ }^{20}$ and Morgan et al. ${ }^{21}$ demonstrated low recurrences (4.3\% and $0 \%$, respectively) when this combination of therapies was applied, but these authors did not specify the follow-up period that was used. The present research demonstrated a CRR of $3.6 \%$ in the 
second year and a CRR of $15.3 \%$ from the third year to the fifth year (Table 2). Despite the methodological differences, all three studies indicated the efficacy of the combination of peripheral ostectomy with the application of Carnoy's solution to control the recurrence of OKC.

The problems attributed to the combination of peripheral ostectomy with the application of Carnoy's solution is the sum of its possible complications. To evaluate the benefit of this association, the present research compared its results with the results observed when only peripheral ostectomy was applied. Despite the lower recurrence in the absence of the Carnoy`s solution (10\%), the RFPs of the two treatments did not demonstrate a significant difference (Table 1). The CRR for the combined treatment $(15.3 \%)$ was similar to that of the peripheral ostectomy $(16.7 \%)$ until the end of the third year, which represented the most critical period for OKC recurrence, as observed in the literature and demonstrated here ${ }^{30,32}$ (Table 2).

Complementary treatments could be compared as an independent variable of decompression, but this was not possible in the present study due to the lack of an adequate sample size of lesions submitted to direct enucleation associated with peripheral ostectomy. However, decompression did not influence the results for such a group because its CRR was acceptable for the first three years and because no recurrence was registered among the decompressed lesions. For the group that underwent the application of Carnoy's solution, one recurrence manifested among the lesions was submitted to decompression.

It can be suggested that the application of Carnoy's solution did not significantly increase the efficacy of peripheral ostectomy. However, the benefits of its use cannot be ruled out, particularly for selected cases. Although peripheral ostectomy did have similar efficacies with and without the application of Carnoy's solution, these data are not applicable to OKCs associated with the NBCCS because $92.3 \%$ of them underwent the combined complementary treatments.

The present study did not display a significant difference for the recurrence of syndromic and non-syndromic lesions, and this is probably due to the effectiveness of the complementary treatments. It must be considered that the good results related to the treatment of syndromic lesions were obtained from only a few subjects who were diagnosed with NBCCS. The low recurrence for the syndromic group can also be related to a less aggressive pattern of the lesions in some of the subjects, which is not in agreement with the current literature.

Donatsky and Hjørting-Hansen ${ }^{33}$ observed a CRR of 55\% at the fifth year for syndromic OKCs that underwent enucleation without complementary treatment. In a recent study with syndromic OKCs, Carlson et al..$^{16}$ demonstrated that enucleation followed by peripheral ostectomy was associated with a CRR of $14 \%$ (or $86 \%$ for no recurrence) at the end of five years. The present study displayed favorable results for the treatment of syndromic OKCs after direct enucleation associated with peripheral ostectomy in combination with the application of Carnoy's solution because the CRR was null for the same period (Table 2 and Figure 8).

Although the five-year recurrence estimate was only positive for the non-syndromic lesions (risk of $25 \%$ ), the results seem to be occasional and not indicative of a higher aggressiveness for such a group of lesions. The absence of significant differences between the RFPs of the treatment methods support this hypothesis and suggest that the analyzed treatments for syndromic and non-syndromic OKCs lesions have similar efficacies.

\section{Conclusion}

Considering the applied methodology and results that are presented here, the following can be assumed: a) decompression does not seem to increase the recurrence risk; b) peripheral ostectomy seemed to demonstrate a similar efficacy when combined with Carnoy's solution, at least for lesions that were not associated with NBCCS; c) the association of NBCCS does not seem to significantly influence OKC recurrence; and d) syndromic lesions seem to behave in the same manner as non-syndromic ones when submitted to complementary treatments. 


\section{References}

1. Shear M. The aggressive nature of the odontogenic keratocyst: is it a benign cystic neoplasm? Part 2. Proliferation and genetic studies. Oral Oncol. 2004;38(4):323-31. https://doi.org/10.1016/\$1368-8375(01)00066-5

2. Gomes CC, Gomez RS. Odontogenic keratocyst: a benign cystic neoplasm? Oral Oncol. 2007;43(6):619-20. https://doi.org/10.1016/i.oraloncology.2006.09.008

3. Li TJ. The odontogenic keratocyst: a cyst, or a cystic neoplasm? J Dent Res. 2011;90(2):133-42. https://doi.org/10.1177/0022034510379016

4. Wright JM, Odell EW, Speight PM, Takata T. Odontogenic tumors, WHO 2005: where do we go from here? Head Neck Pathol. 2014:8(4):373-82. https://doi.org/10.1007/s12105-014-0585-x

5. Pogrel MA. The keratocystic odontogenic tumour (KCOT): an odyssey. Int J Oral Maxillofac Surg. 2015;44(12):1565-8. https://doi.org/10.1016/i.ijom.2015.03.008

6. Pindborg JJ, Kramer JR, Torloni H.. Histological typing of odontogenic tumours, jaw cysts and allied lesions. Geneva: World Health Organization; 1971.

7. Kramer IRH, Pindborg JJ, Shear M, eds. WHO international histological classification of tumours. Histological typing of odontogenic tumours., 2nd ed. Heidelberg: Springer-Verlag; 1992.

8. Forssell K, Sainio P. Clinicopathological study of keratinized cysts of the jaws. Proc Finn Dent Soc. 1979:75(3):36-45. https://doi.org/10.1007/BF02350317

9. Lam KY, Chan ACL. Odontogenic keratocysts: a clinicopathological study in Hong Kong Chinese. Laryngoscope. 2000;110(8):1328-32. https://doi.org/10.1097/00005537-200008000-00020

10. Philipsen HP. World Health Organization classification of tumours. In: Barnes L, Eveson JW, Reichart P, Sidransky D, eds. Title. Lyon: IARC Press; 2005. p. 306-7.

11. Speight P, Devilliers P, Li TJ, Odell EW, Wright JM. World Health Organization classification of tumours. In: El-Naggar AK, Chan JKC, Grandis JR, Takata T, Slootweg PJ, eds. Title. Lyon: IARC Press; 2017. p. 235-6.

12. Brøndum N, Jensen VJ. Recurrence of keratocysts and decompression treatment. A long-term follow-up of forty-four cases. Oral Surg Oral Med Oral Pathol. 1991;72(3): 265-9. https://doi.org/10.1016/0030-4220(91)90211-T

13. Stoelinga PJ. Long-term follow-up on keratocysts treated according to a defined protocol. Int J Oral Maxillofac Surg. 2001;30(1):14-25. https://doi.org/10.1054/ijom.2000.0027

14. Nakamura N, Mitsuyasu T, Mitsuyasu Y, Taketomi T, Higuchi $Y$, Ohishi M. Marsupialization for odontogenic keratocysts: long-term follow-up analysis of the effects and changes in growth characteristics. Oral Surg Oral Med Oral Pathol Oral Radiol Endod. 2002:94(5):543-53. https://doi.org/10.1067/moe.2002.128022
15. Habibi A, Saghravanian N, Habibi M, Mellati E, Habibi M. Keratocystic odontogenic tumor: a 10 -year retrospective study of 83 cases in an Iranian population. J Oral Sci. 2007:49(3):229-35. https://doi.org/10.2334/josnusd.49.229

16. Carlson ER, Oreadi D, McCoy JM. Nevoid basal cell carcinoma syndrome and the keratocystic odontogenic tumor. J Oral Maxillofac Surg. 2015;73(12 Suppl):S77-86. https://doi.org/10.1016/i.joms.2015.04.037

17. Pogrel MA. Decompression and marsupialization as definitive treatment for keratocysts: a partial retraction. J Oral Maxillofac Surg. 2007;65(2):362-3. https://doi.org/10.1016/i.joms.2006.09.032

18. Al-Moraissi EA, Pogrel MA, Ellis E 3rd. Enucleation with or without adjuvant therapy versus marsupialization with or without secondary enucleation in the treatment of keratocystic odontogenic tumors: a systematic review and meta-analysis. J Craniomaxillofac Surg. 2016;44(9):1395-403. https://doi.org/10.1016/j.jcms.2016.05.020

19. Chrcanovic BR, Gomez RS. Recurrence probability for keratocystic odontogenic tumors: an analysis of 6427 cases. J Craniomaxillofac Surg. 2017;45(2):244-51. https://doi.org/10.1016/i.jcms.2016.11.010

20. Chow HT. Odontogenic keratocyst: a clinical experience in Singapore. Oral Surg Oral Med Oral Pathol Oral Radiol Endod. 1998;86(5):573-7. https://doi.org/10.1016/S1079-2104(98)90348-1

21. Morgan TA, Burton CC, Qian F. A retrospective review of treatment of the odontogenic keratocyst. J Oral Maxillofac Surg. 2005;63(5):635-9. https://doi.org/10.1016/i.joms.2004.07.026

22. Leung YY, Lau SL, Tsoi KY, Ma HL, Ng CL. Results of the treatment of keratocystic odontogenic tumours using enucleation and treatment of the residual bony defect with Carnoy's solution. Int J Oral Maxillofac Surg. 2016;45(9):1154-8. https://doi.org/10.1016/j.ijom.2016.02.002

23. Antonoglou GN, Sándor GK, Koidou VP, Papageorgiou SN. Non-syndromic and syndromic keratocystic odontogenic tumors: systematic review and meta-analysis of recurrences. J Craniomaxillofac Surg. 2014;42(7):e364-71. https://doi.org/10.1016/i.jcms.2014.03.020

24. Evans DG, Ladusans EJ, Rimmer S, Burnell LD, Thakker N, Farndon PA. Complications of the naevoid basal cell carcinoma syndrome: results of a population based study. J Med Genet. 1993;30(6):460-4. https://doi.org/10.1136/jmg.30.6.460

25. Bradley PF, Fisher AD. The cryosurgery of bone. An experimental and clinical assessment. Br J Oral Surg. 1975;13(2):111-27. https://doi.org/10.1016/0007-117X(75)90001-3 
26. Stoelinga PJW, Cohen MM Jr, Morgan AF. The origin of keratocysts in the basal cell nevus syndrome. J Oral Surg. 1975;33(9):659-63.

27. Warburton G, Shihabi A, Ord RA. Keratocystic odontogenic tumor (KCOT/OKC): clínical guidelines for resection. J Maxillofac Oral Surg. 2015;14(3):558-64. https://doi.org/10.1007/s12663-014-0732-7

28. Al-Moraissi EA, Dahan AA, Alwadeai MS, Oginni FO, Al-Jamai JM, Alkhutari AS et al. What surgical treatment has the lowest recurrence rate following the management of keratocystic odontogenic tumor?: A large systematic review and meta-analysis. J Craniomaxillofac Surg 2017;45(1):131-44. https://doi.org/10.1016/i.jcms.2016.10.013

29. Forssell K. The primordial cyst: a clinical and radiographic study. Proc Finn Dent Soc. 1980;76(3):129-74.

30. Forssell K, Forssell H, Kahnberg KE. Recurrence of keratocysts. A long-term follow-up study.
Int J Oral Maxillofac Surg 1988;17(1):25-8.

https://doi.org/10.1016/S0901-5027(88)80224-8

31. Gosau M, Draenert FG, Müller S, Frerich B, Bürgers R, Reichert TE et al. Two modifications in the treatment of keratocystic odontogenic tumors (KCOT) and the use of Carnoy's solution (CS): a retrospective study lasting between 2 and 10 years. Clin Oral Investig. 2010;14(1):27-34. https://doi.org/10.1007/s00784-009-0264-6

32. Apajalahti S, Hagstrom J, Lindqvist $C$ and Suomalainen A. Computerized tomography findings and recurrence of keratocystic odontogenic tumor of the mandible and maxillofacial region in a series of 46 patients. Oral Surg Oral Med Oral Pathol Oral Radiol Endod. 2011;111(3):e29-37. https://doi.org/10.1016/j.tripleo.2010.10.010

33. Donatsky O, Hiørting-Hansen E. Recurrence of the odontogenic keratocyst in 13 patients with the nevoid basal cell carcinoma syndrome: a 6-year follow-up. Int J Oral Surg. 1980;9(3):173-9. https://doi.org/10.1016/S0300-9785(80)80016-0 